\title{
Rheological yield stress measurement of paste fill: New technical approaches
}

\author{
Maria Silva \\ Somincor, Lundin Mining Corporation, Portugal \\ Martin Hansson \\ Sika Tunnelling and Mining, Sweden \\ Matilde Costa e Silva \\ Cerena, Técnico, University of Lisbon, Portugal
}

SUMMARY: Paste backfill technology has become increasingly relevant to the mining industry, providing not only a safe way for the underground disposal of tailings but also essential advantages for ground stabilization and optimized ore recovery. Yield stress is among the most important rheological properties of paste backfill, which determines its transportability during long distances. Measurement of yield stress is a challenging and complex task, given the high number of variable factors. So far, no standard procedures and methods have been established for measuring the rheological properties of paste backfill, in particular yield stress.

This experimental work consists of the development of an accurate laboratory testing programme that will allow for the evaluation, measurement and understanding of rheological yield stress of paste fill. For this study, tailings from Zinkgruvan (Sweden) and Neves-Corvo (Portugal) mines were analysed. A series of laboratorial tests were conducted including the following test procedures: slump, flow table spread, fall cone and the vane technique (applied using a viscometer and a rheometer).

The correlations between the yield stress, measured by vane technique and other test methods, were obtained from the test results. Additionally, preliminary conclusions were drawn regarding the influence of physical properties of tailings (particle size distribution, dry content, uniformity coefficient and coefficient of gradation) on yield stress by a statistical study using multiple linear regression models.

The fall cone test has resulted in the best correlation measurement of dry content and of yield stress measurements using the viscometer and rheometer. Being a simple, inexpensive, and expedited method for paste yield stress measurements, it is considered effective for quality control and/or rapid on-site measurements of paste fill.

Keywords: Paste Fill, Yield Stress, Rheology, Fall Cone

\section{INTRODUCTION}

With increasing public pressure to handle mine waste material more carefully, paste backfilling has proven to be the most important creation of the last 30 years, because it significantly reduces the disposal of surface tailings, by placing them safely in underground stopes (Yilmaz and Fall, 2017). Aside from providing a safe way for storing tailings in underground mined-out stopes, 
paste backfill also has essential advantages for ground stabilization, such as: providing a safer work environment for miners, working platform, and enabling the exploitation of adjacent stopes (increasing ore recovery).

The major challenges associated with this increasingly popular technology in underground mines are related to the massive consumption of binder and its associated costs, including the efficient/reliable transport of freshly mixed paste to the mined-out stopes. In order to optimize the paste mix design, a maximized solid content is desired while maintaining a pumpable consistency. However, high solid mixes often create increased densities during transport, in particular as the mine advances into greater depth and the reticulation system increases in length.

Technological advancements in thickening, filtration, centrifugation, mixing and pumping ability are all dependent upon an increased understanding of paste flow behaviour/workability and strength characteristics (Sofra, 2017).

\subsection{Paste fill rheology}

Rheology is among the most important properties of paste fill materials. Understanding the rheological properties of a paste fill material is essential to predict its behaviour during mixing, pumping, transport and curing.

Paste fill normally exhibits pseudoplastic behaviour with a yield stress, and in some instances, thixotropic behaviour.

Yield stress is the critical shear stress that must be exceeded before irreversible deformation and flow can occur. This property is considered by many authors as the most important rheological parameter for the design and operation of a paste system. Physical and chemical/mineralogical properties of paste fill have a significant influence on yield stress, such as: solids content, percentage of cement, particle size distribution (PSD), particle shape, mineralogy, surface properties of tailings, among others.

In terms of PSD, the quantity of fine particles present is very important for transportation because fine particles help to float the coarse grains in the paste and provide a non-settling paste flow within the network of pipes (Ercikdi et al, 2017). As a rule of thumb, a minimum of $15 \%$ of particles having a diameter lower than $20 \mu \mathrm{m}$ (fines fraction) is considered sufficient to generate water retention properties required for the transport of tailings paste through a borehole/pipeline system (Landriault, 1995).

Particle morphology or shape can have dramatic effects on rheology, particularly when plate or needle-like particles with high aspect ratios are present.

The mineralogy of tailings influences a number of paste backfill features, such as water retention, strength, settling characteristics and abrasive action (Ercikdi et al. 2017). However, it is not clear how it specifically influences the rheological behaviour of paste. The chemical composition of tailings is of utmost importance for the mechanical strength development of paste backfill.

The precise yield stress, as a true material constant, has turned out to be very difficult to measure, as different tests often give out different results: "depending on the measurement geometry and the detailed experimental protocol, very different values of the yield stress may be found" (James et al, 1987; Nguyen \& Boger, 1992; Barnes, 1997,1999; Barnes \& Nguyen 2001).

Consequently, there is a need to standardise procedures for yield stress measurements and for the conditioning/preparation of paste samples in order to achieve significant improvements in mine backfill (Silva, 2017).

\section{EXPERIMENTAL WORK}

The experimental work consists of the development of an accurate laboratorial testing program to evaluate, measure and understand the rheological behaviour and properties of fresh paste fill. To this effect, a series of experimental tests were conducted at GEOLAB - Instituto 
Superior Técnico in Lisbon, using different paste fill mixtures. These were produced using Portland cement type II A/L 42.5R, tap water and tailings from Zinkgruvan (Sweden) and Neves-Corvo (Portugal) mines, subsidiaries of Lundin Mining Corporation. Both tailing samples were collected following the filtration process (filter cake) and were kept inside 45-litres barrels.

An optimal interval of solid content was set for each mine tailings, where the laboratorial tests could produce valid results and the material would behave like a real mix at the mine site.

To reduce the number of variables, the percentage of cement was set at $4 \%$ and, for each mine, three mix designs were established.

The Neves-Corvo (NC) tailings were used to produce mixtures of $78 \%, 80 \%$ and $82 \%$ of solids. In the case of Zinkgruvan (ZG) tailings, these presented different physical properties, visible on PSD curves. For barrels 1 and 2 the mixtures had $78 \%, 79 \%$ and $80 \%$ of solids, while for barrels 3 and 4 , the mixtures made had $79 \%, 80 \%$ and $81 \%$ of solids.

\subsection{Physical and chemical characterization of mine tailings}

The physical and chemical characterization of tailings was conducted by the Corporate Analytic Service of Sika Technology in Zürich, Switzerland.

The particle size distribution (PSD) analysis was determined using a laser diffraction technique. Each sample was manually sieved with a mesh size of $1 \mathrm{~mm}$ to separate big agglomerates. The cumulative and density distributions of NC and ZG tailings are shown in Figure 1. The curves correspond to each barrel of tailings.

ZG tailings are much coarser than NC tailings, showing an accentuated slope of the curve starting in $20 / 30 \mu \mathrm{m}$ of particle size. In terms of the differences between barrels, the PSD curves for NC barrels are equals, which proves that they were collected at the same time. In contrast, the PSD curves of ZG barrels show a clear difference between barrels $1 / 2$ and 3/4, suggesting that the tailings were collected on separate days. It is also possible that barrel 4 may have not been collected at the same time as barrel 3, due to a slight difference in the maximum values of the curves.

Given this, only NC barrel 1 (NC1) and ZG barrels 1 and 3 (ZG1 and ZG3) samples were considered for particle size, chemical composition and mineralogy. These were determined by scanning electron microscopy (SEM) with energy dispersive $\mathrm{x}$-ray analysis (EDS).

Based on SEM images, it is noticeable that ZG tailings have more angular and elongated particles than NC tailings (Figure 2).

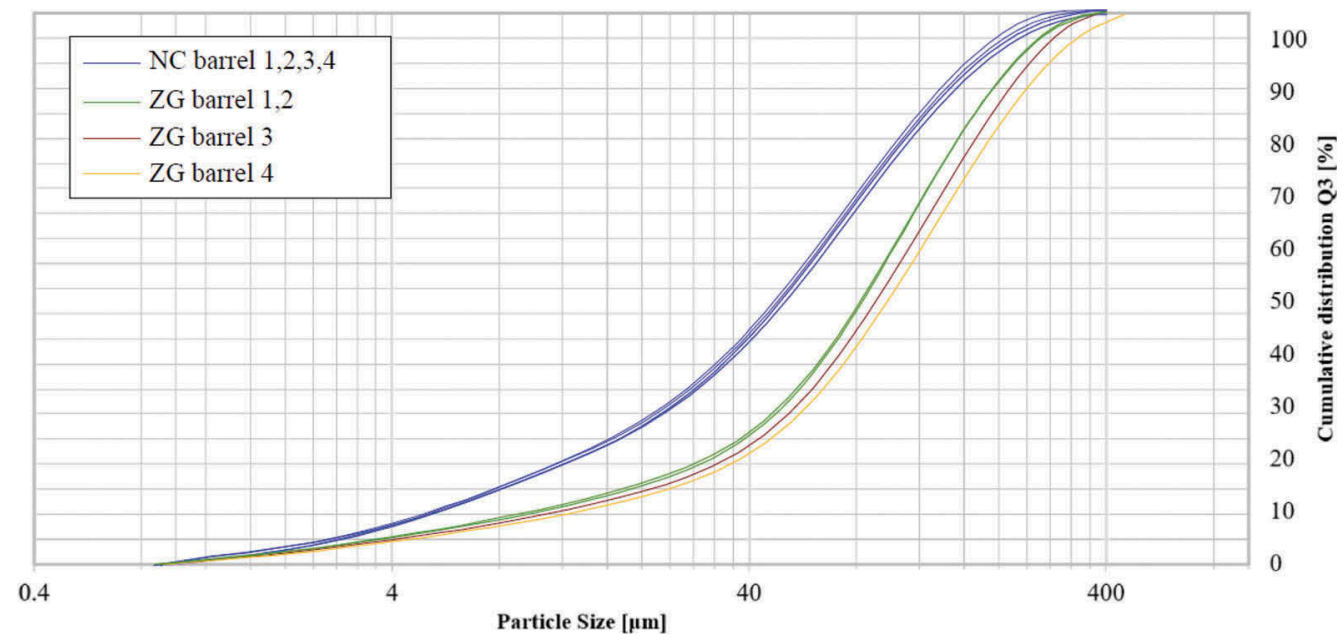

Figure 1. Cumulative distributions of NC tailings (on top) and ZG tailings (on bottom). 

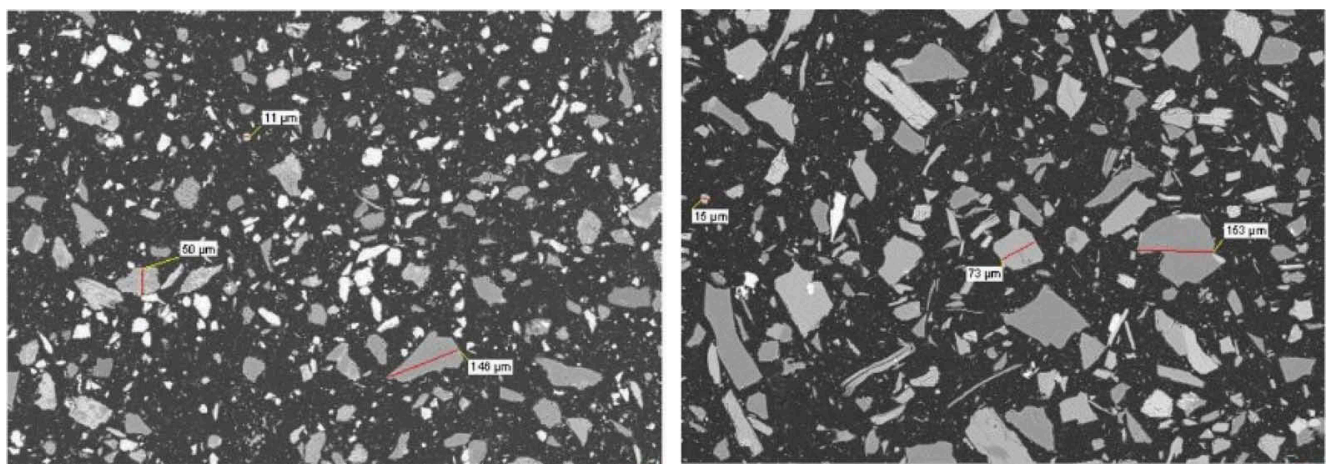

Figure 2. SEM images of samples NC1 and ZG3.

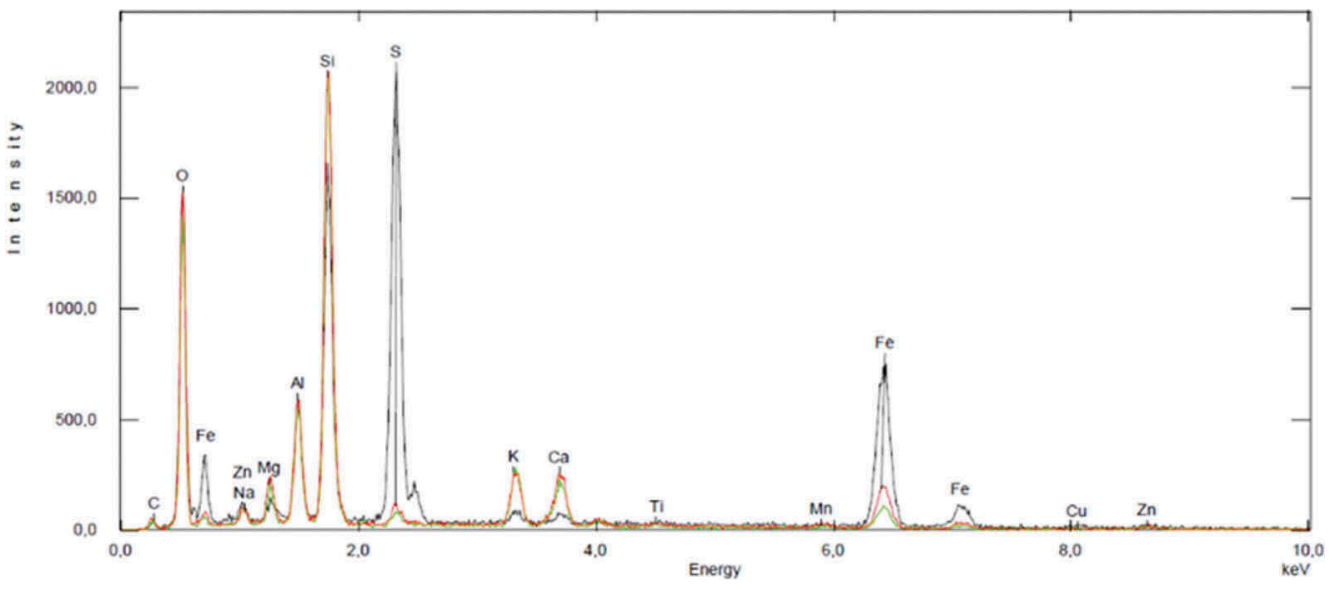

Figure 3. EDS spectra comparison of the samples NC1 (black), ZG1 (red) and ZG3 (green).

In terms of chemical composition, the Bulk EDS spectra presented in Figure 3 clearly shows a much higher sulphur and iron content in NC tailings, as well as a higher silica and alkali content in $\mathrm{ZG}$ tailings.

\subsection{Sample preparation}

The tailings from each barrel were divided into samples of approximately $4 \mathrm{~kg}$ and sealed in plastic bags to prevent evaporation.

To measure the water content of each sample/bag, the tailings were mixed for homogenization by a Hobart mixer and then a small specimen, was taken, weighted and dried in the oven for 24 hours at $80^{\circ} \mathrm{C}$.

Knowing the water content and total weight of the sample, it was allowed to calculate the quantity of cement and water to be added to produce the desired mix.

The mixing procedure combined different rotational speeds, agitators and mixing times. First, the tailings were placed inside the mixer bowl and mixed for 1 minute using the flat beater at speed 1 . The whip attachment then replaced the flat beater and the water was slowly added at speed 1. After 1 minute of mixing, the cement was slowly added. At last, the final mixture was prepared at speed 2 for 3 minutes to ensure that the materials were well blended by the Hobart mixer. 


\subsection{Test procedure}

Mixing the trials were then undertaken in the following order: dry content, bulk density, viscometer, rheometer, fall cone (with $30^{\circ}$ and $60^{\circ}$ ), flow table and slump.

The procedure adopted for bulk density is similar to standards ASTM C138/C 138M-01a and BS EN 12350-6 2009. Standard SCAN-P 39:80 was followed for determining the dry content.

The slump test was carried out using a mini cone with a bottom diameter of $100 \mathrm{~mm}$, a top diameter of $50 \mathrm{~mm}$ and a height of $150 \mathrm{~mm}$. The procedure adopted was similar to standards EN 12350-2 and ASTM C143/C 143M-05a, used for concrete.

To measure the slump-flow, using a flow table for cement mortar, a cone with a bottom diameter of $100 \mathrm{~mm}$, a top diameter of $70 \mathrm{~mm}$ and height of $50 \mathrm{~mm}$ was used. The procedure adopted was similar to standard ASTM C230, used for cement mortar. The diameter of the paste was measured twice: the first measure was taken after the cone lifting and the second measure after the 15 strokes made with the up and down movement of the table.

The absolute values of yield stress were determined by vane technique, widely accepted by many authors as the best method for achieving the "true" yield stress: "the great advantage of the vane is the material yields on itself this technique is now used worldwide for measurement of yielding in a variety of materials and was motivated by the need to understand the true yielding behaviour under plant/site conditions" (Sofra, 2017). This technique was applied in a controlled shear rate mode (stress growth technique) by a viscometer and a rheometer with vane spindles.

The viscometer used was the DV1 Brookfield rotational viscometer, model RV, with fourblade vanes (V73 and V75) and the Wingather SQ software, V4.0.7. This equipment was programmed to a constant speed of $0.3 \mathrm{rpm}$ and a running time of 2 minutes and 15 seconds.

The rheometer used was the Anton Paar RheolabQC instrument with the vane spindle ST22-6V-16 and the Rheoplus software V3.62. In this case, the rheometer was programmed to a constant speed of $0.1 \mathrm{rpm}$ and a running time of 3 minutes and 20 seconds.

In an attempt to obtain a practical and inexpensive test method to measure the yield stress onsite, some experiments were carried out with a Controls S.R.L fall cone instrument, using a standard metal cone of 30 degrees. This method is well known in soil mechanics for measuring the liquid limit and the undrained shear strength of fine-grained soils.

Due to the weight of the cone, it became wholly immersed at every attempt. As such, the exact geometry of the metal cone and bar had to be recreated and attached using a lighter material. After some unsuccessful reproductions, two final prototypes were printed in a Formlabs 3D printer, at the maximum definition $(0.025 \mathrm{~mm})$ with a special resin for top quality output, durability and toughness. The cones were printed with 30 and 60 degrees, weighing $12 \mathrm{~g}$ and $18 \mathrm{~g}$, respectively (Figure 4). The procedure is quite simple. A container was filled with paste and the top surface levelled with a spatula. The cone tip resting on the paste surface is released and, following penetration, it is stopped as quickly as possible. The depth of penetration is then recorded. The containers used were $53 \mathrm{~mm}$ in diameter and a $68 \mathrm{~mm}$ in height for the $30^{\circ}$-cone, and $85 \mathrm{~mm}$ in diameter and $57 \mathrm{~mm}$ in height for the $60^{\circ}$-cone.

\section{RESULTS}

A total of 72 mixtures were produced and the number of values recorded were in excess of 500. The average of all results is presented in Tables 1 and 2.

The shear stress-time curves and the correlation graphs between the yield stress and the dry content of ZG and NC mixtures are shown in Figures 5, 6 and 7.

Throughout all figures, it is possible to identify the domains of each mix design, especially in NC charts. These domains are mainly due to the differences in solids concentration between mixtures, being the dry content interval higher in $\mathrm{NC}$ than in ZG mixtures. With exception to the ZG curves, the residual shear stresses also show very perceptible domains achieved by the rheometer. It is possible to observe that, after yielding, the curves recover some of the stress 

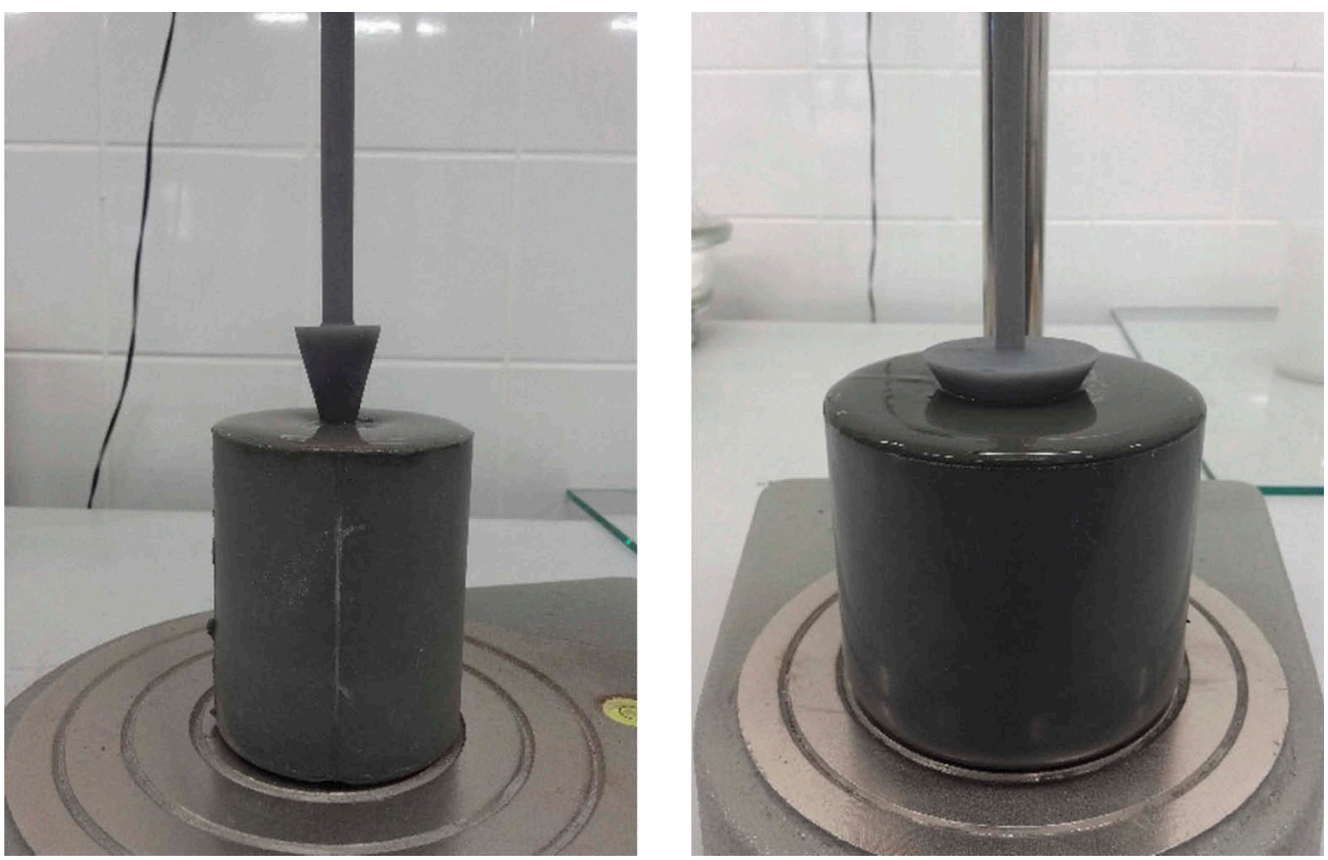

Figure 4. Printed cones with 30 and 60 degrees and respective containers.

Table 1. Experimental result of $Z \mathrm{Z}$ mixtures.

\begin{tabular}{|c|c|c|c|c|c|c|c|c|c|}
\hline \multicolumn{10}{|c|}{ ZINKGRUVAN MIXTURES } \\
\hline \multirow[b]{2}{*}{$\begin{array}{l}\text { Solid } \\
\text { content } \\
{[\%]}\end{array}$} & \multirow[b]{2}{*}{$\begin{array}{c}\text { Dry } \\
\text { Content } \\
{[\%]}\end{array}$} & \multirow[b]{2}{*}{$\begin{array}{l}\text { Density } \\
{\left[\mathrm{kg} / \mathrm{m}^{3}\right]}\end{array}$} & \multicolumn{2}{|c|}{ Vane Technique } & \multicolumn{2}{|c|}{ Flow Table } & \multicolumn{2}{|c|}{ Fall Cone } & \multirow[b]{2}{*}{$\begin{array}{l}\text { Slump } \\
{[\mathrm{cm}]}\end{array}$} \\
\hline & & & $\begin{array}{l}\text { Yield stress } \\
\text { (viscometer) } \\
{[\mathrm{Pa}]}\end{array}$ & $\begin{array}{l}\text { Yield stress } \\
\text { (rheometer) } \\
{[\mathrm{Pa}]}\end{array}$ & $\begin{array}{l}\text { Initial } \\
\mathrm{D}[\mathrm{cm}]\end{array}$ & $\begin{array}{l}\text { Final } \\
\mathrm{D}[\mathrm{cm}]\end{array}$ & $\begin{array}{l}30^{\circ} \\
\text { Cone } \\
{[\mathrm{mm}]}\end{array}$ & $\begin{array}{l}60^{\circ} \\
\text { Cone } \\
{[\mathrm{mm}]}\end{array}$ & \\
\hline $78 \%$ & 73.47 & 2046.9 & 45.27 & 92.50 & 13.17 & 24.08 & 32.97 & 25.82 & 11.4 \\
\hline $78 \%$ & 73.61 & 2060.97 & 48.65 & 134.67 & 12.17 & 24.08 & 31.2 & 22.7 & 10.93 \\
\hline $79 \%$ & 74.88 & 2020.03 & 55.17 & 167.67 & 10.58 & 23.42 & 25.77 & 19.83 & 9.5 \\
\hline $79 \%$ & 74.66 & 2030.27 & 54.31 & 217.33 & 10.58 & 22.5 & 23.73 & 18.67 & 9.5 \\
\hline $79 \%$ & 75.07 & 2052.17 & 65.06 & 256.67 & 10.63 & 22.13 & 22.8 & 19.47 & 8.4 \\
\hline $79 \%$ & 74.7 & 2020.17 & 62.81 & 225.67 & 10.5 & 22.58 & 24 & 19.83 & 7.33 \\
\hline $80 \%$ & 75.59 & 1983.09 & 62.85 & 353.33 & 9.67 & 21.67 & 20.27 & 16 & 6.67 \\
\hline $80 \%$ & 75.71 & 2034.88 & 80.62 & 245.67 & 9.58 & 20.08 & 16.8 & 14.27 & 6.77 \\
\hline $80 \%$ & 76.77 & 2080.5 & 93.32 & 377 & 9.75 & 18 & 11.67 & 10.8 & 5.43 \\
\hline $80 \%$ & 75.64 & 2033.6 & 85.60 & 281.33 & 9.58 & 19.33 & 15.23 & 14.23 & 6.67 \\
\hline $81 \%$ & 76.61 & 2027.41 & 86.69 & 349 & 9.5 & 18.33 & 14.5 & 12.27 & 5.9 \\
\hline $81 \%$ & 76.95 & 1997.49 & 98.94 & 373.33 & 9.5 & 17.83 & 13.03 & 11.03 & 5.93 \\
\hline
\end{tabular}

and, therefore, the residuals are not distributed by domains/intervals as shown in NC curves. This suggests that ZG paste fill could be time-dependent for shearing at low shear rates (most probably thixotropic behaviour). However, additional tests should be undertaken to prove and understand this rheological behaviour.

The test methods applied in this experimental work are well known in the mining industry for evaluating the rheology of concrete/paste backfill, particularly the slump test. There are 
Table 2. Experimental result of NC mixtures.

\begin{tabular}{|c|c|c|c|c|c|c|c|c|c|}
\hline \multicolumn{10}{|c|}{ NEVES-CORVO MIXTURES } \\
\hline \multirow[b]{2}{*}{$\begin{array}{l}\text { Solid } \\
\text { content } \\
{[\%]}\end{array}$} & \multirow[b]{2}{*}{$\begin{array}{l}\text { Dry } \\
\text { Content } \\
{[\%]}\end{array}$} & \multirow[b]{2}{*}{$\begin{array}{l}\text { Density } \\
{\left[\mathrm{kg} / \mathrm{m}^{3}\right]}\end{array}$} & \multicolumn{2}{|c|}{ Vane Technique } & \multicolumn{2}{|c|}{ Flow Table } & \multicolumn{2}{|c|}{ Fall Cone } & \multirow[b]{2}{*}{$\begin{array}{l}\text { Slump } \\
{[\mathrm{cm}]}\end{array}$} \\
\hline & & & $\begin{array}{l}\text { Yield stress } \\
\text { (viscometer) } \\
\text { [Pa] }\end{array}$ & $\begin{array}{l}\text { Yield stress } \\
\text { (rheometer) } \\
{[\mathrm{Pa}]}\end{array}$ & $\begin{array}{l}\text { Initial } \\
\mathrm{D}[\mathrm{cm}]\end{array}$ & $\begin{array}{l}\text { Final } \\
\mathrm{D}[\mathrm{cm}]\end{array}$ & $\begin{array}{l}30^{\circ} \\
\text { Cone } \\
{[\mathrm{mm}]}\end{array}$ & $\begin{array}{l}60^{\circ} \\
\text { Cone } \\
{[\mathrm{mm}]}\end{array}$ & \\
\hline $78 \%$ & 77.29 & 2374.51 & 114.15 & 184.33 & 14.33 & 23.25 & 31.7 & 22.13 & 12.17 \\
\hline $78 \%$ & 76.86 & 2396.11 & 78.16 & 177 & 17.83 & 24.92 & 35.43 & 25.07 & 12 \\
\hline $78 \%$ & 76.91 & 2369.63 & 84.03 & 187.33 & 16 & 23.83 & 33.67 & 22.37 & 11.83 \\
\hline $78 \%$ & 76.79 & 2378.35 & 68.61 & 190.33 & 17.75 & 25.17 & 34.87 & 24.27 & 12.5 \\
\hline $80 \%$ & 79.05 & 2598.43 & 177.32 & 381.67 & 10.25 & 20.75 & 23.2 & 16.33 & 9.5 \\
\hline $80 \%$ & 78.84 & 2416.48 & 170.12 & 491.33 & 10 & 21.17 & 25.23 & 18.87 & 8.83 \\
\hline $80 \%$ & 79 & 2422.93 & 167.48 & 449.33 & 9.92 & 20.58 & 23.73 & 16.4 & 8.33 \\
\hline $80 \%$ & 78.77 & 2433.95 & 160.87 & 480.33 & 10.75 & 21.67 & 26.6 & 16.6 & 10.33 \\
\hline $82 \%$ & 81.21 & 2515.84 & 245.93 & 1023.5 & 9.125 & 16.125 & 10.4 & 9.15 & 4.75 \\
\hline $82 \%$ & 80.95 & 2458.19 & 339.95 & 1360 & 9.17 & 17.75 & 12.3 & 9.3 & 5 \\
\hline $82 \%$ & 80.73 & 2483.49 & 364.48 & 1553.33 & 9.25 & 17.08 & 10.87 & 8.13 & 4.33 \\
\hline $82 \%$ & 80.84 & 2515.63 & 340.68 & 1680 & 9.25 & 18.67 & 13.6 & 9.8 & 4.5 \\
\hline
\end{tabular}
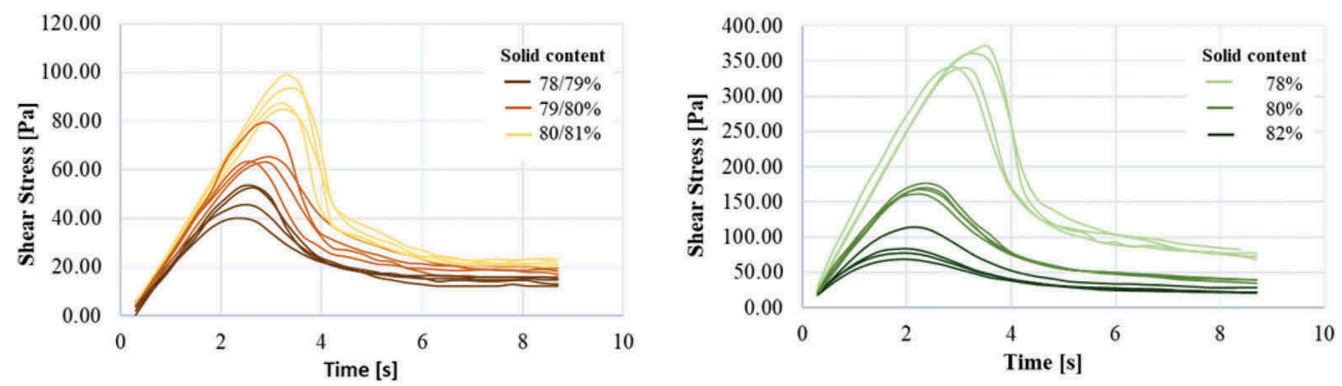

Figure 5. Shear stress-time curves for ZG (left) and NC (right) mixtures as recorded by the viscometer.
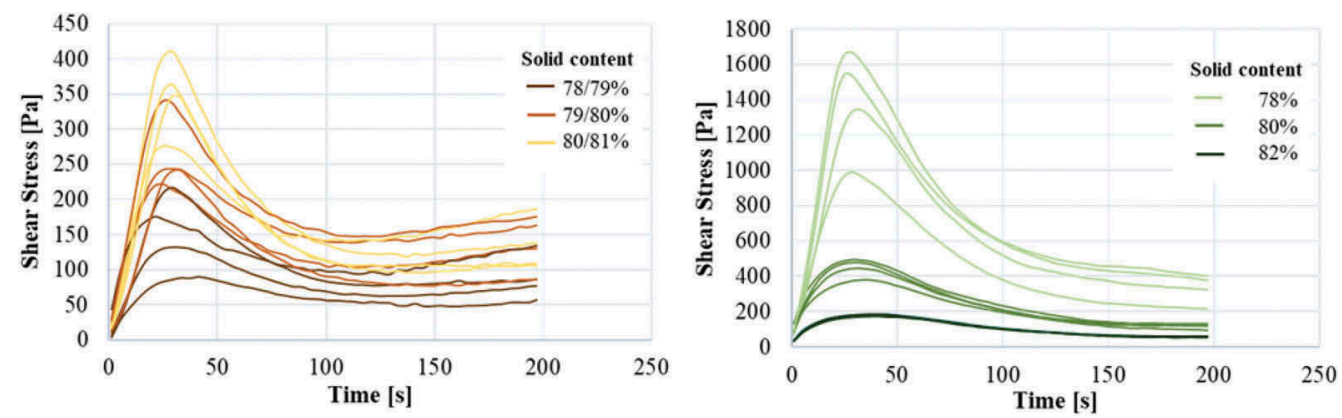

Figure 6. Shear stress-time curves for ZG (left) and NC (right) mixtures as recorded by the rheometer.

formulations that allow us to determine the yield stress of samples from the results of these tests. However, the purpose of this work was to evaluate the fitting capacity of these methodologies to different paste fill mixtures, with different tailings and mix designs, and to determine the veracity and quality of the results and their correlations with the yield stress values measured by a rheometer and a viscometer. 

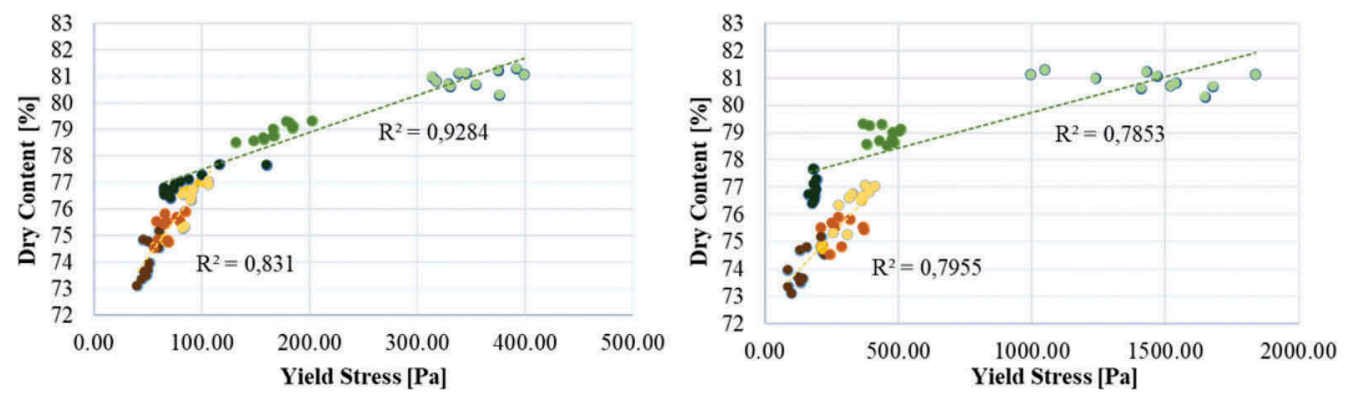

Figure 7. Correlation graphs of yield stress recorded by the viscometer (left) and rheometer (right) versus dry content of mixtures.

From the test method results, the measurement that achieved the worst performance was the initial diameter of flow table. It was not able to characterize mixtures with higher dry contents using this measurement, because the diameter of the cone mould is identical.

The correlation graphs between the results obtained by the slump, fall cone and flow table spread (final diameter) were correlated with the dry content and the absolute yield stress (measured by the viscometer and rheometer) of all mixtures are presented in Figures 8 and 9.

\section{RESULTS DISCUSSION}

The mix designs set for each mine tailings, during the initial run practice, were different in terms of workability range. This range was smaller for ZG mixtures $(78-80 \%$ and $79-81 \%$ solids content) than for NC mixtures ( $78 \%-82 \%$ solids content), mainly due to the physical properties of both tailings.
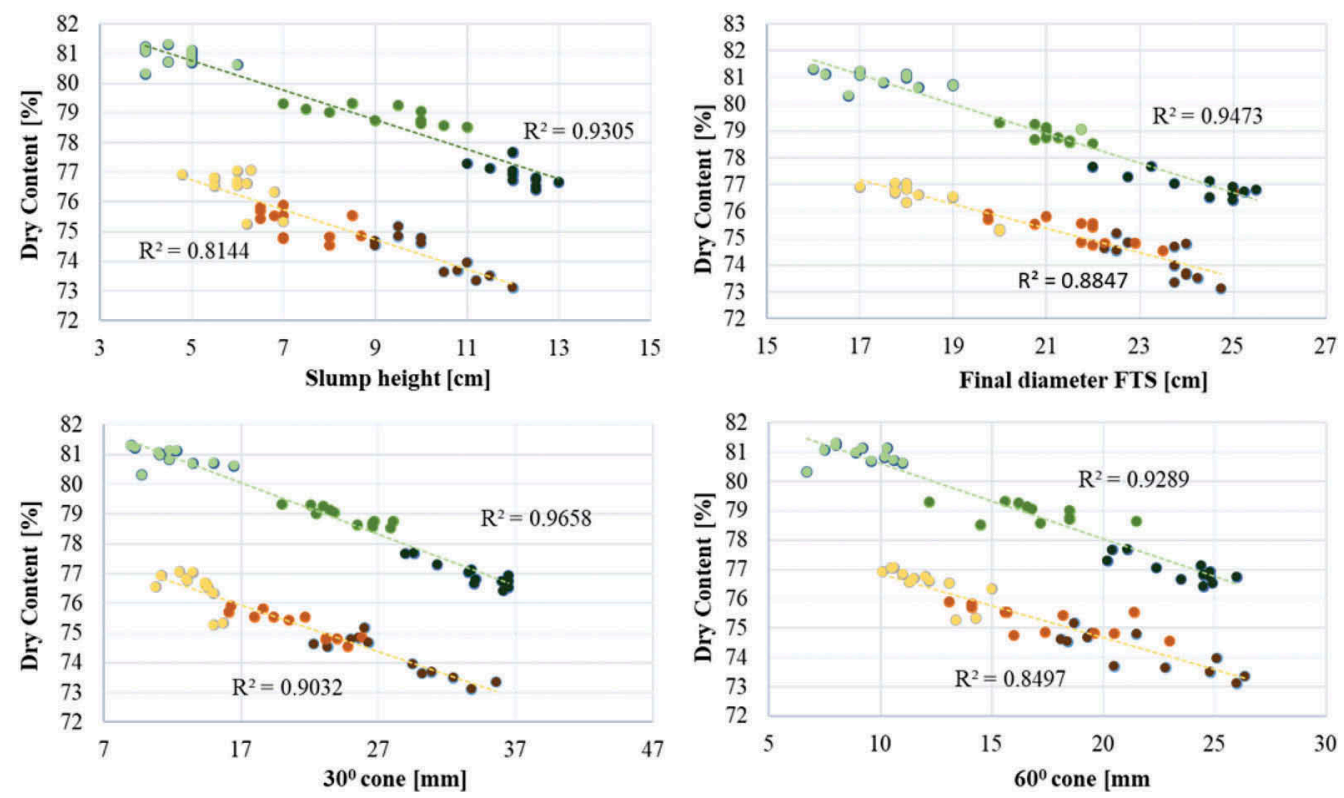

Figure 8. Correlations graphs between the dry content and tests' results. ZG in orange and NC in green. 

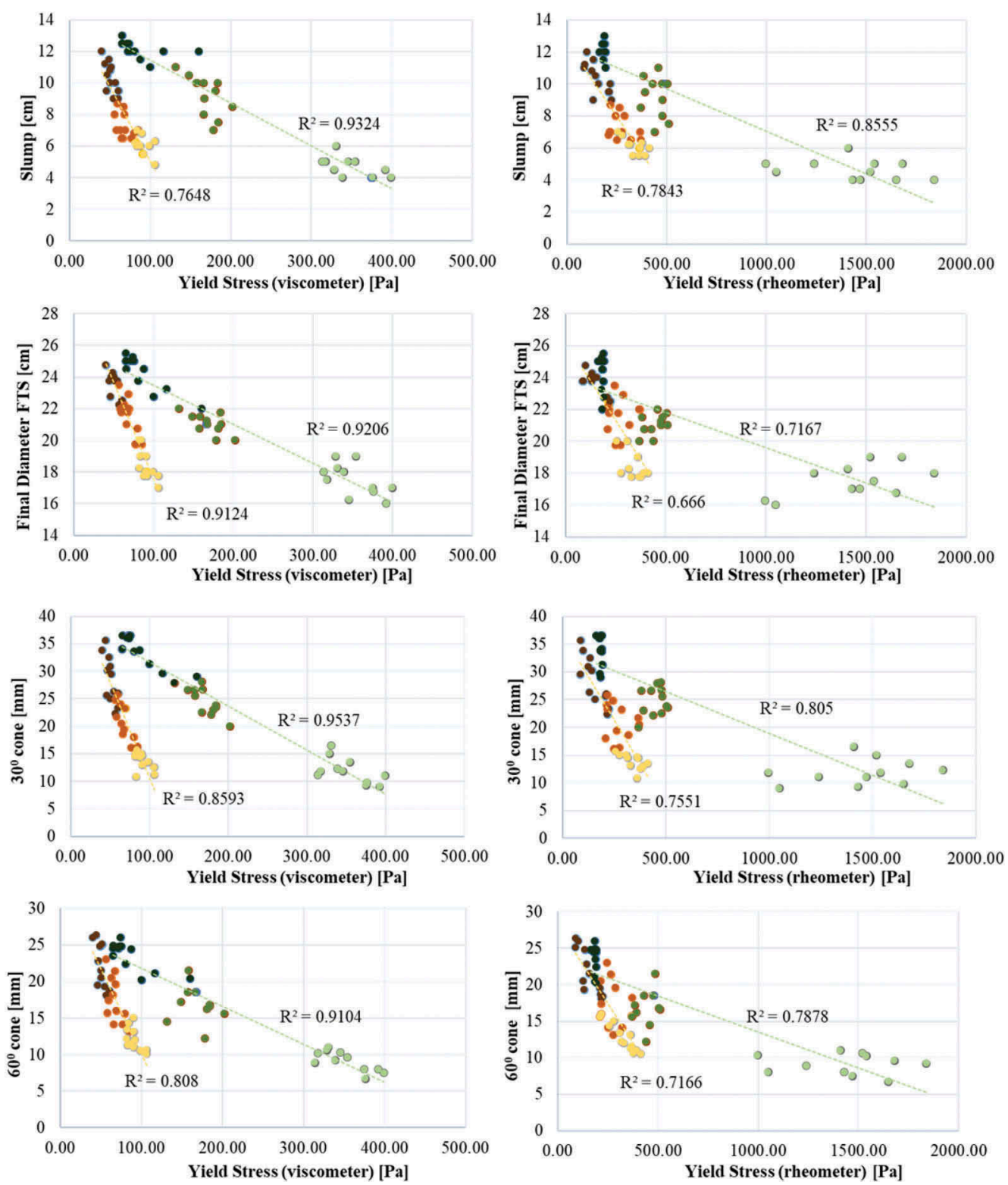

Figure 9. Correlations graphs between the yield stress recorded by the viscometer and rheometer and the tests' results. ZG in orange and $\mathrm{NC}$ in green.

In general, finer slurries generally display a more gradual increase in yield stress with increasing solids concentration, whereas coarse materials show a relatively sharp transition from liquid-like to solid-like behaviour (Sofra, 2017), which may justify the smaller interval of workability for ZG paste fill when compared with a finer material such as NC tailings, using the same cement percentage and water.

When observing Tables 1 and 2, the dry content values are consistent for each mix design. Another noticeable effect is the density difference of both paste mixtures, which can be explained by the increase in solids concentration of the mixtures but, moreover, because of the 
tailings' mineralogy. $\mathrm{NC}$ tailings contain denser elements $\left(\mathrm{Fe}_{2} \mathrm{O}_{3}, \mathrm{SO}_{3}\right.$, etc.) than $\mathrm{ZG}$ tailings $\left(\mathrm{SiO}_{2}, \mathrm{Al}_{2} \mathrm{O}_{3}\right.$, etc. $)$.

The absolute yield stress values were measured using the viscometer and the rheometer. The rheometer results were less consistent than the values measured by the viscometer. This can be explained by the higher precision of the equipment and lower rotational speed, making it particularly susceptible to slight deviations of the vane spindle from the centre of the container, to existent air bubbles, small agglomerations of particles, wall slip and other factors. Another aspect is related to the dimensions of the container utilised for these measurements: the distance from the vane spindle to the edge of the container was too small. Previous projects that involved the use of a vane in a cup to minimise slip (Barnes and Carnalli, 1990) and infinite medium analysis (Kreiger and Maron, 1954) have proven the major advantages of using the vane in an infinite medium for this type of measurement, such as (Sofra, 2017): insertion of a vane causes less sample disturbance, minimizing thixotropic breakdown; the vane in cup is less susceptible to errors arising from large particle sizes; yielding occurs between layers of fluid; minimizing the effects of wall slip, among others. Since ZG tailings are coarser than NC material, the presence of larger particles and more air voids causes more disturbance during the rotation of the vane spindle. This could be the reason for the inconsistent results using ZG tailings.

Due to the higher rotational speed of the viscometer, the values of yield stress are smaller. The dimensions of the container were high enough compared to the diameter of the vane spindle, creating an infinite medium for measuring paste rheology. Both mine mixtures presented consistent results and behaviours.

In general, it is worth noting that $\mathrm{NC}$ mixtures present higher yield stress values in comparison to ZG mixtures for the same solids' concentration, suggesting that the dry content could not be the only variable responsible for the variance of yield stress. In this view, the physical and chemical differences of both tailings can also be influencing factors. In terms of the influence of tailings mineralogy on rheological properties of paste fill, only general information is available in academic works. Therefore, it is, in this case, difficult to conclusively assess which parameters most affect the rheology of paste backfill in terms of chemical composition. In general, as NC mixtures are denser than those from ZG, due to their mineralogy, the shear necessary to start flowing is naturally higher.

From the test method results, the measurement that presented the worst performance was the initial diameter of flow table. Apart from this, all methods were able to evaluate mix design differences and obtain a good correlation with yield stress values.

The final diameter proved to be a perfect measurement of paste fill rheology. The data was consistent and the correlations with yield stress values were even better in comparison to the slump test (Figures 10 and 11).

Finally, the adapted fall cone test turned out to be the methodology that performed the best, with very good correlation with the dry content of the mixtures and with the yield stress values achieved by the viscometer and rheometer (Figure 9 and 10). The weight and geometry of the cones combined with the time released proved to be a successful procedure (Figure 12) for measuring paste fill rheology.

As mentioned before, this test method is widely used in soil mechanics to obtain the undrained shear strength of fine-grained soils, based on the "cone factor", $K$. Reports from different authors show $K$ values ranging from 0.4-1.33. Differences are mostly attributed to the cone surface roughness (Llano et al., 2018).

To compare the $K$ factor of the $30^{\circ}$-cone used for this experimental work with other standard cones, this was calibrated following the procedure proposed by Llano et al., 2018. To this effect, the yield stress values measured by the viscometer that presented the best correlations with the fall cone were used. All the results obtained for both mixtures were applied.

A correlation graph with the yield stress and the $Q / h^{2}$ was drawn, where $Q$ is the total cone weight and $h$ is the cone penetration depth. The slope of the best fit straight lines corresponded to the $K$ factor. This varies between $0.26-0.39$. 


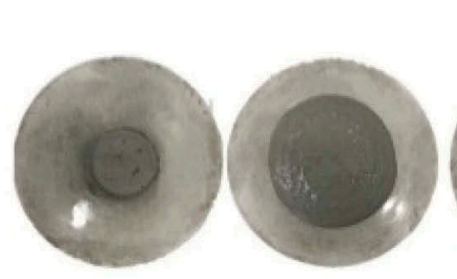

$74.15 \%$

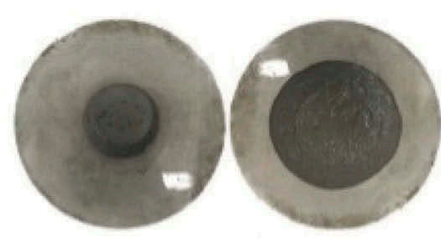

$81.18 \%$

ZINKGRUVAN PASTE FILL

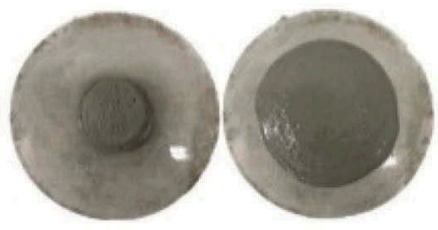

$75.26 \%$

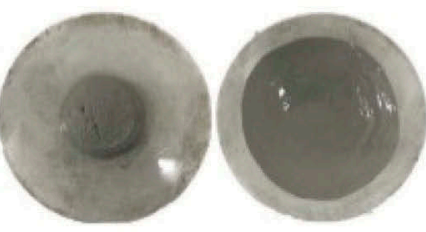

$74.15 \%$

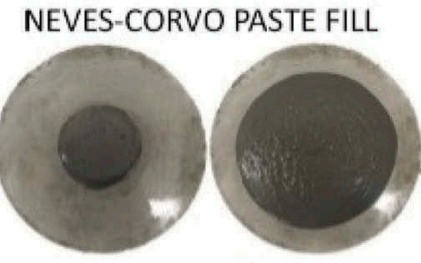

$78.92 \%$

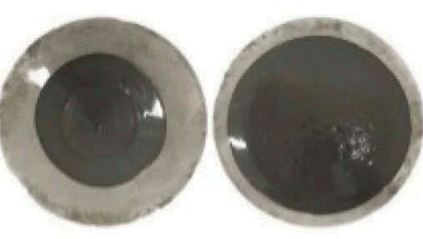

$76.96 \%$

Figure 10. Photographic record of flow table tests.

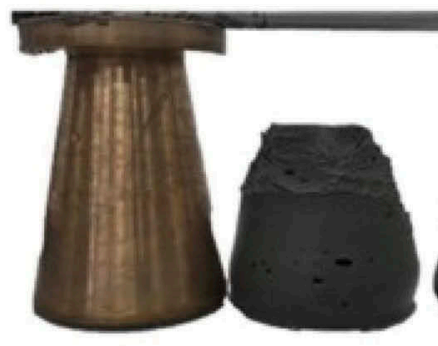

$76.49 \%$

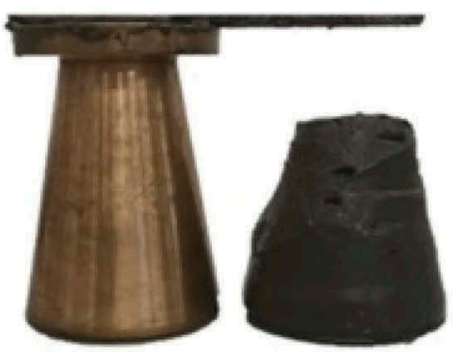

$81.18 \%$

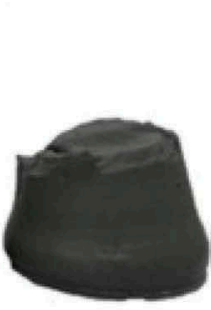

$75.26 \%$

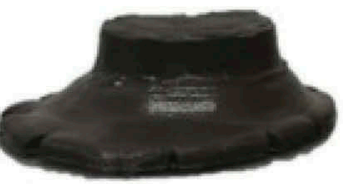

$78.92 \%$
ZINKGRUVAN

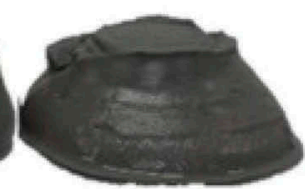

$74.15 \%$

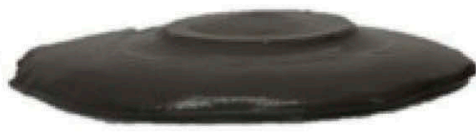

$76.96 \%$

Figure 11. Photographic record of slump tests.

These $K$ interval are admissible in the range values for the $30^{\circ}$ standard cones. However, this is a conservative estimation and for greatest accuracy, further values should be taken.

\subsection{Data modelling}

As previously concluded, the dry content of both mixtures was not the only influencing property on yield stress results. In an attempt to mathematically prove the influence of the physical 


\section{ZINKGRUVAN PASTE FILL}
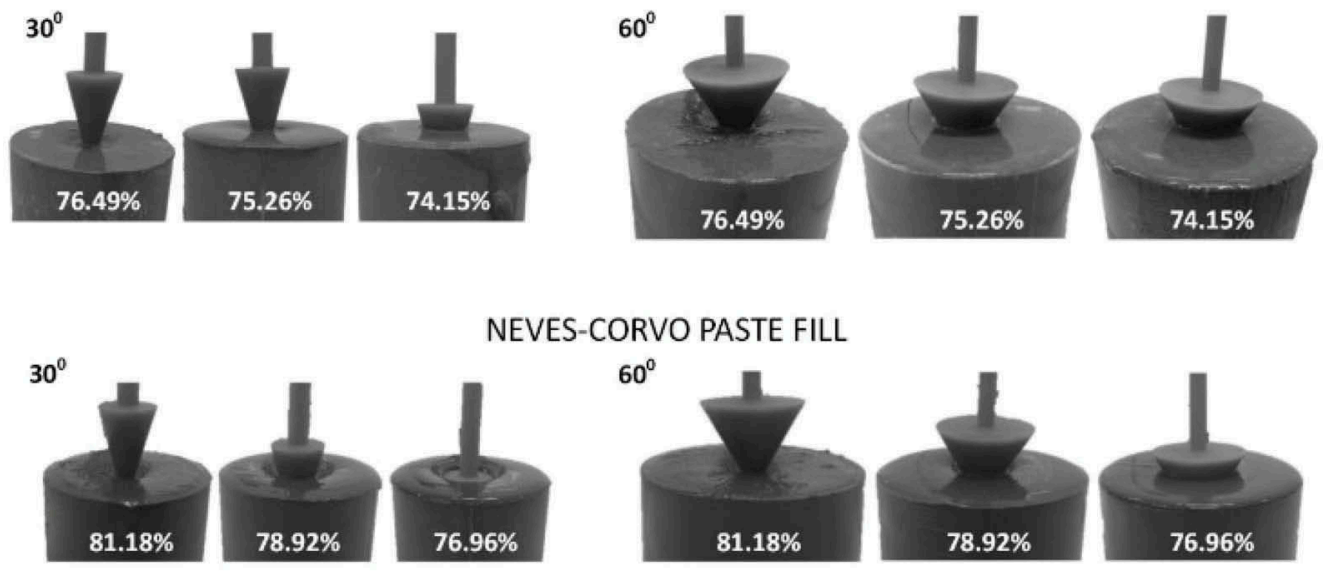

Figure 12. Photographic record of fall cone tests.

properties tailings on yield stress, two detailed statistical studies were developed. They are based on the proposal and validation of multiple linear regression models to predict yield stresses measured by both equipment (dependent variable) in all mixtures, as a function of the following quantitative predictors: dry content, percentage of material passing under 20 micron $(<20 \mu \mathrm{m})$, uniformity coefficient $\left(C_{u}\right)$ and coefficient of gradation $\left(C_{c}\right)$. The remaining characteristic diameters of PSD were not considered because they are applied in the calculation of coefficients $C_{u}$ and $C_{c}$ (Silva, 2017).

The validation of each candidate model included the statistical significance of the estimated parameters, the suitability of the adjusted model by analysis of variance (decomposition of the total sum of squares of the residuals) and the statistical quality of the regression model through residual analysis (Paneiro et al., 2015). In this sequence, the quality of the models was assessed using the following criteria: coefficients of determination $\left(R^{2}\right.$ and $R^{2}$ adjusted), analysis of variance (ANOVA table) and Akaike and Schwarz-Bayesian information criteria (AIC and SBIC).

Considering the laboratorial data and statistical analysis, the best multiple linear regression models can be written as follows (Equations 1 and 2):

$$
\begin{aligned}
\log \left(\text { yield stress }_{\text {visc }}\right)=4.6571 & +0.7482 \log (\text { DryC })+0.1724 \log \left(C_{C}\right)-0.2201 \log \left(C_{u}\right) \\
+0.2374 \log (<20 \mu \mathrm{m}) & \\
\log \left(\text { yield stress }_{\text {rheo }}\right) & =5.8085+1.0157 \log (\text { DryC })+0.1816 \log \left(C_{C}\right) \\
& -0.3180 \log \left(C_{u}\right)-0.5957 \log (<20 \mu \mathrm{m})
\end{aligned}
$$

It is worth noting that yield stress $_{\text {visc }}$, yield stress $_{\text {rheo }}, \operatorname{Dry} C, C_{C}, C_{u}$ and $<20 \mu m$ correspond to normalized variables.

The models presented very high adjusted coefficients of determination ( 0.9570 and 0.8937 , respectively) and rejected the null hypothesis by f-test statistics analysis, which shows a very good level of explanation of the laboratorial data variability (Silva, 2017).

Based on these models, it is possible to conclude that the dry content of mixtures as well as the particle size of tailings are strong influencing variables in yield stress values determined using a viscometer and rheometer. However, it is necessary to consider other influencing variables such as mineralogy/chemical composition that may have a significant influence on yield 
stress. Furthermore, the weight of each quantitative predictor is questionable. To validate these models, a higher number of results and other influencing parameters should be analysed.

\section{FINAL CONSIDERATIONS AND RECOMMENDATIONS}

Rheology can play an essential role in better understanding and dealing with thickened tailings. Due to the necessity of creating a standard experimental testing program to evaluate paste fill worldwide, a simple testing procedure using a drop cone measurement was developed.

Paste mixtures were produced using tailings from Zinkgruvan and Neves-Corvo mines, 4\% of cement and tap water. To determine the absolute values of yield stress, the vane technique with a viscometer and a rheometer at different rotational speeds was applied. The results obtained showed that the range of yield stress for both mine mixtures was different. From the properties assessed, the dry content of mixtures was the main variable that affected this rheological property.

The laboratory program included the following test methods: dry content, bulk density, viscometer, rheometer, fall cone (with $30^{\circ}$ and $60^{\circ}$ ), flow table spread and slump. Of all laboratory test methods, the least convincing measurement was the initial diameter of the flow table. Apart from this, all test methods enabled the assessment of the difference between mix designs and obtained a good correlation between the yield stress measured by both pieces of equipment. The flow table (final diameter) and the $30^{\circ}$ fall cone presented the best performances.

To verify the influence of the physical properties of tailings on yield stress, two detailed studies based on the proposal and validation of multiple linear regression models were developed. The models obtained have shown a strong correlation of tailings' physical properties on yield stress. However, these must be validated with more results and other influencing factors such as mineralogy/chemical composition included in future works.

The fall cone test adopted for this experimental work turned out to be the methodology of choice that performed best, with a good correlation of dry content and the yield stress values achieved by the viscometer and rheometer. The weight and geometry of the cones combined with the release time have proven to be a successful procedure for measuring paste fill rheology, and thus represent one of this work's major contributions. This equipment can become a simple, inexpensive, convenient and fast method for determining yield stress, ideal for quality control and/or fast on-site measurements.

\section{ACKNOWLEDGEMENTS}

This research was sponsored by Sika Corporation (Switzerland R\&D Department, Sweden, Portugal and Spain), and we would like to personally thank Fabian Erismann, Christophe Kurz and Rute Silva for their guidance and assistance during the project.

We are grateful to Zinkgruvan and Neves-Corvo mines for providing the material and equipment necessary for the laboratorial tests.

We gratefully acknowledge Rodolfo Machado and Hugo Brás for sharing their experience and knowledge, which greatly supported the elaboration of the laboratorial testing programme developed in this experimental work.

\section{BIBLIOGRAPHY}

Barnes, H. A. 1997. Thixotropy - a review. Journal of Non-Newtonian Fluid Mechanics 70, pp. 1-33.

Barnes, H.A. 1999. The yield stress - a review or ' $\pi \alpha \nu \tau \alpha \rho \varepsilon \mathrm{i}^{-}$- everything flows? Journal of NonNewtonian Fluid Mechanics 81, pp. 133-178. 
Barnes, H.A. and Carnali J.O. 1990. The vane-in-cup as a novel rheometer geometry for shear thinning and thixotropic materials. The Journal of Rheology 34(6), pp. 841-866.

Barnes, H. A. and Nguyen, Q. D. 2001. Rotating vane rheometry - a review. Journal of Non-Newtonian Fluid Mechanics 98, pp. 1-14.

Ercikdi et al. 2017 - Ercikdi, B., Cihangir, F., Kesimal, A. and Deveci, H. 2017. Practical Importance of Tailings for Cemented Paste Backfill. Yilmaz, E. and Fall, M. (eds) Paste Tailings Management, Springer, Cham, pp. 7-32.

James, A. E., Williams, D. J. A. and Williams, P. R. 1987. Direct measurement of static yield properties of cohesive suspensions. Rheologica Acta 26, pp. 437-446.

Kreiger, I.M. and Maron, S.H. 1954. Direct determination of the flow curves of non-Newtonian fluids, III. Standardised treatment of viscometric data. Journal of Applied Physics 25 (1), pp.72-75.

Landriault, D. 1995. Paste backfill mix design for Canadian underground hard rock mining. Proceedings of the $97^{\text {th }}$ annual general meeting of the CIM rock mechanics and strata control session, pp. 652-663.

Llano-Serna, M. A., Farias, M. M., Pedroso, D. M., Williams, D. J. and Sheng, D. 2018. Considerations on the Experimental Calibration of the Fall Cone Test. Geotechnical Testing Journal 41 (6), pp.1131-1138

Nguyen, Q.D. and Boger, D.V. 1992. Measuring the flow properties of yield stress fluids. Annual Review of Fluid Mechanics 24, pp. 47-88.

Paneiro, G., Durão, F., Costa e Silva, M. and Neves, P. 2015. Prediction of ground vibration amplitudes due to urban railway traffic using quantitative and qualitative field data. Transportation Research $D$ (40), pp. 1-13.

Silva, M.A. 2017. Contribution to laboratorial determination of rheological properties of paste backfill. Lisbon: IST Press, $82 \mathrm{pp}$.

Sofra, F. 2017. Rheological Properties of Fresh Cemented Paste Tailings. Yilmaz, E. and Fall, M. (eds) Paste Tailings Management, Springer, Cham, pp. 33-57.

Yilmaz, E. and Fall, M. 2017. Introduction to Paste Tailings Management. Yilmaz, E. and Fall, M. (eds) Paste Tailings Management, Springer, Cham, pp. 1-6. 